\title{
Uniform Inbuilt Wireless Sensor Node for Working Conditions Monitoring
}

\author{
Denis Spirjakin, Alexander M. Baranov \\ Moscow Aviation Institute \\ (National Research University), \\ Moscow, Russia \\ Email: denis.spirjakin@gmail.com
}

\begin{abstract}
All companies strive to eliminate work accidents. But still a lot of professions are exposed to different hazardous conditions and many work injuries and even deaths occur everyday. Monitoring of working conditions is a very important part of labor protection. Combining wireless sensor networks with wearable technology is possible to significantly improve safety delivery capability of such systems and add new functionality to them. In this work we present the design results of the uniforms inbuilt wireless sensor node for working conditions monitoring. The node is able to signalize about employee presence in relation to working facilities, and to monitor the atmosphere for temperature and combustible gases concentration. It consists of light-weighted distributed pieces which are built in to clothes and has low power consumption. The average power consumption of the node is low enough for several weeks autonomous lifetime.
\end{abstract}

\section{INTRODUCTION}

$\mathrm{W}$ hile safety standards for industrial plants become tougher, the demand for continuous monitoring of employee state and working conditions including environmental conditions is rising. At the same time modern wearable technology tends to build electronics in to clothes [1] providing new quality in healthcare [2], [3], military [4] and other spheres.

Variety of wireless sensor networks were developed recently. These networks consist of small nodes and are equiped with transceivers, microprocessors and sensors [5], [6]. They can be used in different areas of life (security, military, home automation, etc.). But the most frequent area is environmental [7] and human [8] monitoring.

Incorporating wireless sensor networks with wearable technology is possible to significantly improve safety delivery capability of monitoring systems and add new functionality to them. Such "smart" uniforms can provide insitu monitoring of people, facilities and environmental conditions and in case of an emergency situation assist special services and employees.

It is expected that in the nearest future wireless sensor networks will connect computer networks and physical world. That will lead to tight integration of real and virtual worlds [9] where communication will go between people and devices - Internet of Things [10].

In this work we present the design results of the uniform inbuilt wireless sensor node for working conditions monitoring. The node is able to control employee presence in relation to working facilities, and to monitor the atmosphere for temperature and combustible gases concentration. It consists of light-weighted distributed pieces which are built in to clothes and has low power consumption. The average power consumption of the node is low enough for several weeks autonomous lifetime.

The paper is organized as follows: at first we overview the node in Section II. In Section III we describe the sensing circuit of the node. Section IV is dedicated to data transmission and presence detection principles which are used in this work. The power consumption of the node is discussed in Section V. Finally, we provide concluding remarks in Section VI.

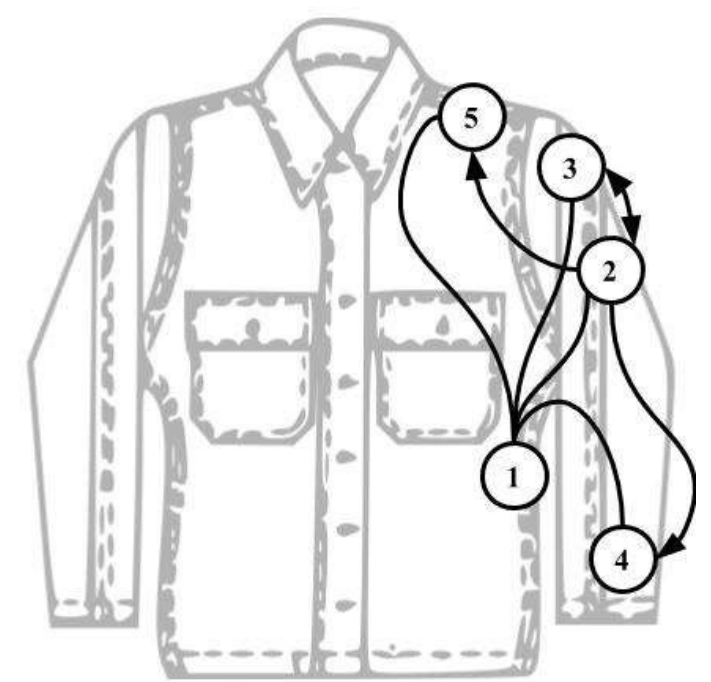

Fig. 1. Block diagram of the node: a power source (1), a sensor module (2), a trasceiver (3), a light indicator (4) and a buzzer (5). 


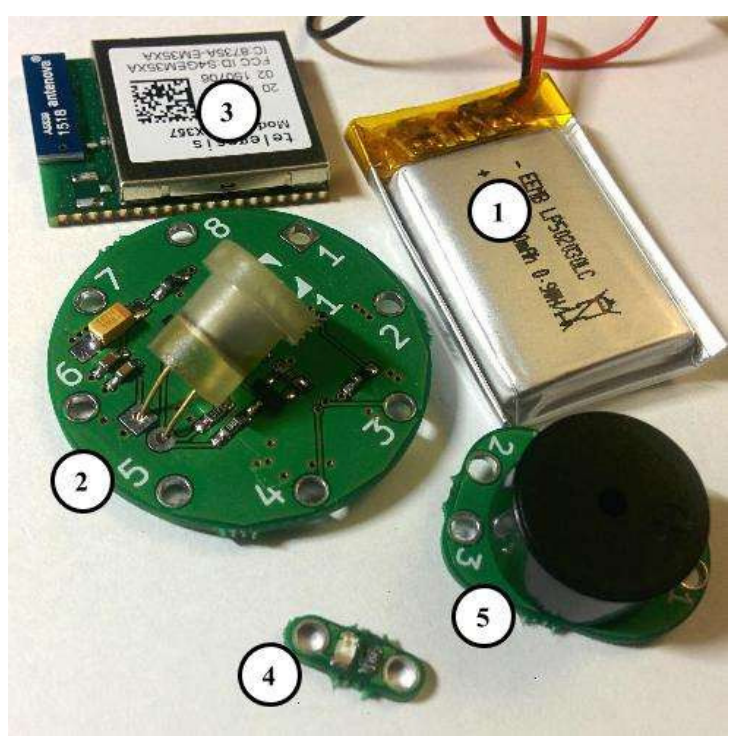

Fig. 2. The node pieces: a power source (1), a sensor module (2), a trasceiver (3), a light indicator (4) and a buzzer (5).

\section{Node Overview}

The diagram of the uniform inbuilt wireless sensor network node is presented in Fig. 1. The node consists of several distributed pieces wich are built in to a jacket: a power source (1), a sensor module (2), a trasceiver (3), a light indicator (4) and a buzzer (5). The node pieces are presented in Fig. 2.

Since the node is wearable and built in to clothes, all its pieces are light and small. At the same time, the pieces are placed according to their functions and distributed around the clothes to make wearing comfortable.

The power source of the node is a lithium-polymer battery. Its nominal voltage is $3.6 \mathrm{~V}$ and the capacity is $250 \mathrm{mAh}$. The weight of the battery is about $5 \mathrm{~g}$ and the dimensions are $30 \times 20 \times 5 \mathrm{~mm}$. The battery is placed in the bottom of the jacket and connected to a ribbon cable to provide power to other pieces.

The sensor module provides sensing of the atmosphere temperature and combustible gases concentration. It also controls the light indicator and the buzzer to maintain alarm signals and send and receive data using the transceiver.

This module is based on ATxmega16E5 microcontroller. It uses built-in temperature sensor to measure the atmosphere temperature and the commercial catalytic gas sensor for combustible gases concentration measuring. The gas sensor is manufactured by NTC IGD (Russia) and its power consumption is $200 \mathrm{~mW}$ in continuous measurement mode. Therefore, the catalytic gas sensor is very power hungry. To decrease its power consumption, the special measuring algorithm is used. More details about the measuring circuit and the algorithm are presented in Section III.

The sensor module is placed on the left shoulder and connected to the power supply ribbon cable and cables to the

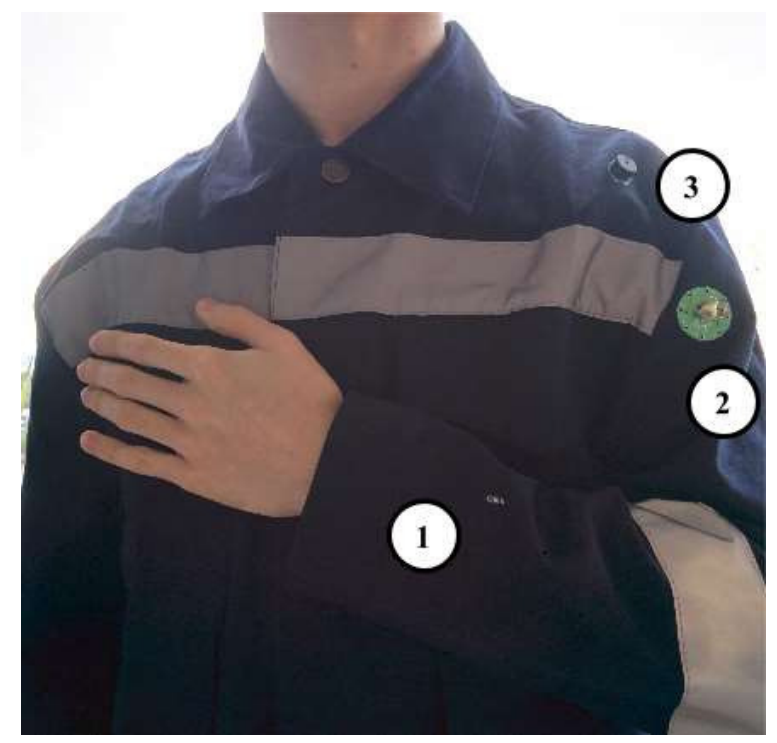

Fig. 3. The assembled node: the light indicator (1), the sensor module (2) and the buzzer (3).

light indicator, the buzzer and the transceiver. The light indicator consists of light emitting diode and current limiting resistor. It placed on the left arm to be on the line of sight of the wearer. The buzzer is placed on the left shoulder near the neck. The task of light indicator and the buzzer is to perform visual and audial alarm signals to wearer when dangerous condition occurs.

Except visual and audial signals, information about dangerous conditions is transmitted to other devices in a wireless sensor network. For this purpose the transceiver module Telegesis ETRX3 is used. The module provides IEEE 802.15.4/Zigbee standards compatible protocol and is controlled by UART interface using AT-style commands set.

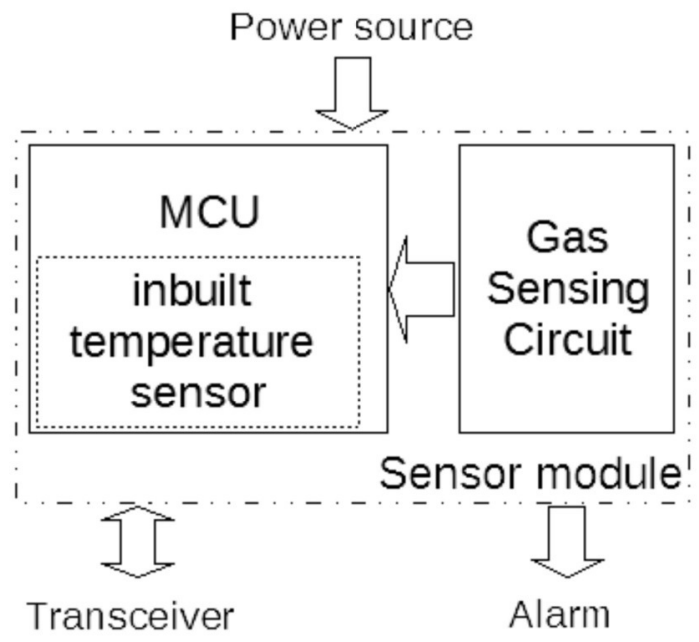

Fig. 4. Block diagram of the sensor module. 


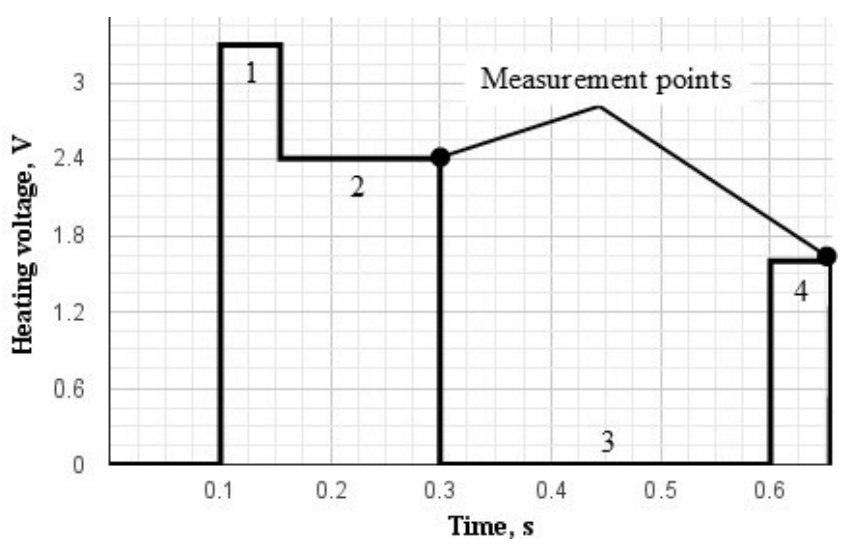

Fig. 5. Gas sensor heating profile.

The transceiver is also used to perform employee's presence tracking. The algorithm to detect wearer presence is described in Section IV.

The node which is assembled on the jacket is presented in Fig. 3.

\section{Sensor Module}

Sensor module consists of the MCU with inbuilt temperature sensor and the gas sensing circuit. To measure gas concentration the catalytic sensor is used. The block diagram of the sensor module is presented in Fig. 4.

Catalytic gas sensor circuits are usually based on the Wheatstone bridge circuit. That circuit consists of two resistors and two sensors, one active and one reference. Measurement process includes heating of the sensors (up to $450 \mathrm{C}$ for methane) which is the main part of sensor's power

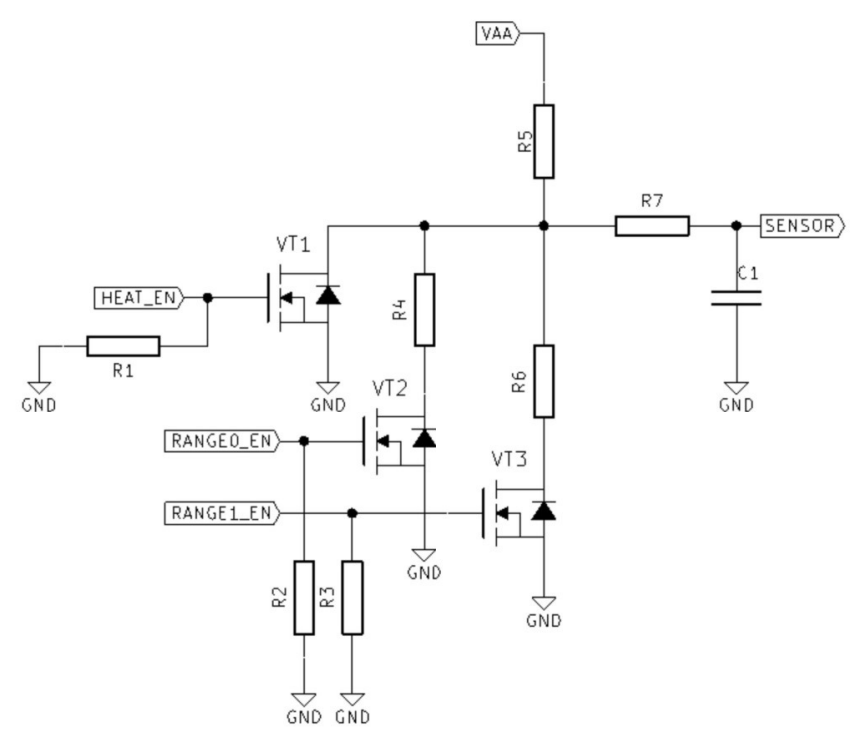

Fig. 6. The gas sensor measuring circuit for the multistage pulse method with PWM heating. consumption. The power consumption value of the Wheatstone bridge circuit is about $200 \mathrm{~mW}$. That's too much and not appropriate for autonomous operation of the node.

Excluding the reference sensor decreases the power consumption. The compensation of the atmosphere humidity and temperature which is usually performed by the reference sensor in this case is fulfilled by using the specific multistage heating pulse. This method was offered and discussed in [11]-[13].

In this work we use four stages for every multistage pulse (1-4 regions in the diagram, Fig. 5).

In the first and the second stages the sensor is heated up to the catalysis external diffusion region (about 450C) and the partial evaporation of surface water is performed. During the third stage heating is not applied. In the final fourth stage the sensor is heated up to the beginning of the catalysis kinetic region (about 200C). After this pulse, the element cools down to ambient temperature. Heating voltages for the stages are $3.3 \mathrm{~V}, 2.4 \mathrm{~V}, 0 \mathrm{~V}$ and $1.6 \mathrm{~V}$ respectively.

The measurement result is the difference between sensor voltages at two different temperatures (measurement points in the diagram).

The gas sensor measuring circuit for the multistage pulse method is presented in Fig. 6. The circuit is controlled by the microcontroller and consists of a sensor R5, a MOSFET key VT1 which controls heating, two range switching MOSFET keys VT2 and VT3 which scale up output signal based on sensor resistance, and an output low pass filter R7-C1 which is connected to microcontroller's inbuilt ADC.

Different voltage levels of the multistage heating pulse are generated by applying pulse-width modulated signal to the heating key. As it was shown in [14] PWM heating allows to significantly decrease sensor power consumption and therefore use a catalytic gas sensor in battery powered autonomous devices.

Range switching keys maintain the scale of circuit output signal inside the input range of ADC converter. Two ranges are used since two measurements per cycle are performed.

To match safety standards [15], [16] the measuring pulses are performed once per 20 seconds. Between pulses to spare the power the node is switched to sleep mode.

Table I. Reliable service area versus transceiver output power.

\begin{tabular}{|r|r|}
\hline Output power, $\mathbf{d B m}$ & Distance, $\mathbf{m}$ \\
\hline-43 & 0.4 \\
\hline-26 & 3 \\
\hline-20 & 5 \\
\hline-17 & 7 \\
\hline-14 & 10 \\
\hline
\end{tabular}




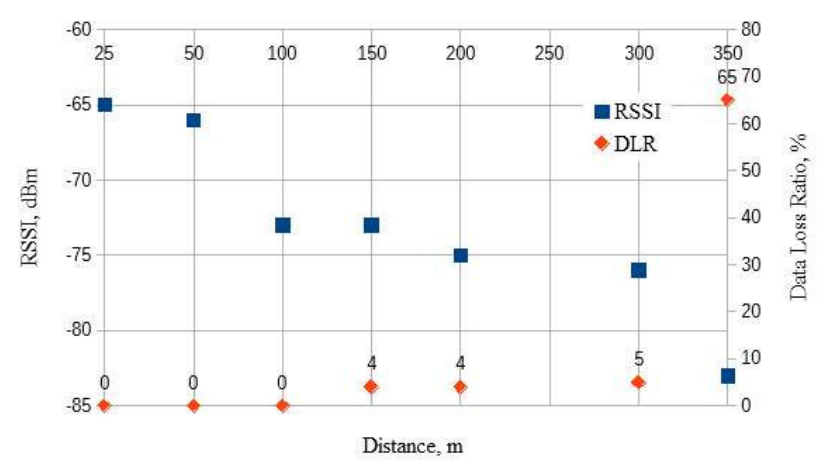

Fig. 7. Relative signal strength versus transmission distance and related data loss ratio.

The node is configured to activate alarm based on two threshold values of methane concentration which are 0.5 and $1 \%$ vol. If the concentration is less than first threshold, there is no relation from the node. If the concentration is going higher than $0.5 \%$ vol. methane, the node provides the light and short sound alarm. When the concentration is more than $1 \%$ vol. the sound alarm becomes longer. In both cases the alarm signal is also sent over the wireless network to the data sink node.

\section{Data Transmission and Presence Detection}

Data transmission is performed by using the transceiver module Telegesis ETRX3. The module is IEEE 802.15.4/Zigbee compatible. The communication with module is performed by UART interface using AT-style command set.

To communicate by wireless network the node should join it before. After it is joined, it has parent node where the data will be sent to later routing. If the connection with parent

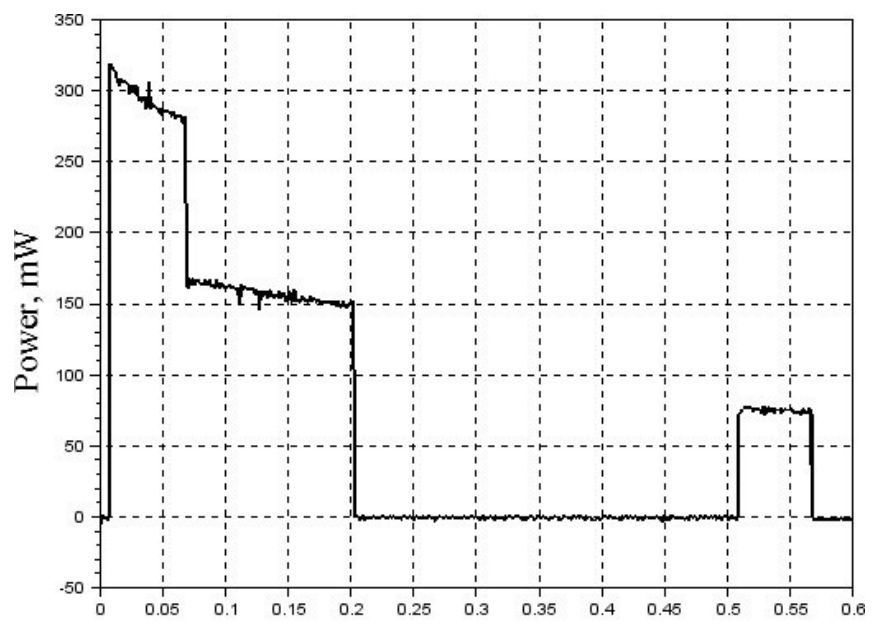

Time, s

Fig. 8. The node power consumption during measurements.

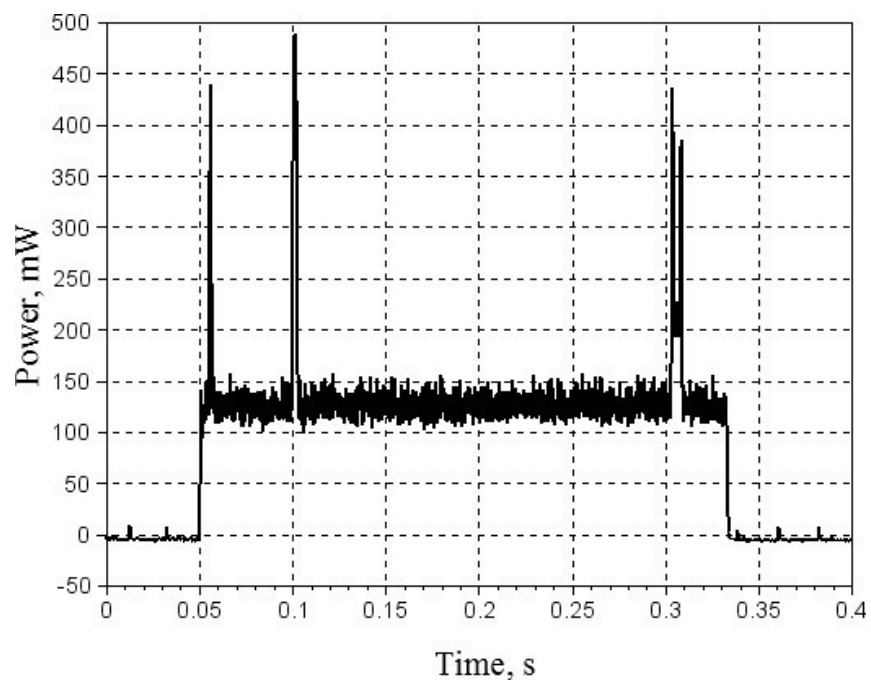

Fig. 9. The node power consumption during data transmission.

node is lost, the node should rejoin the network to be able to send any messages.

The transmission distance depends on the signal power. The transceiver module is able to change its output power. Therefore, the signal transmission distance can be regulated. This feature is used to implement the node presence detection.

Since the node is connected to the network its presence is already detected by parent node. If the connection is lost the node is trying to rejoin the network every work cycle which is 20 seconds. Attempts are continued till the node is joined the network and at that moment the presence will be detected by new parent node.

The detection radius is based on the transmission power of the node. Reliable service distances versus transceiver output power are shown in Table I. The service area is considered as

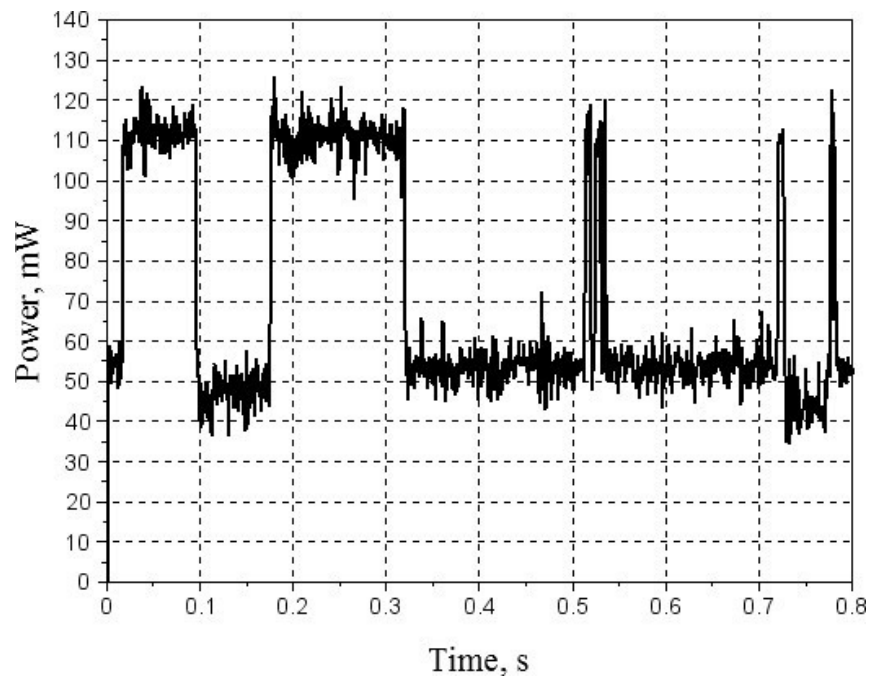

Fig. 10. The node power consumption during joining the network. 
reliable if the RSSI value is more than $-70 \mathrm{dBm}$ which corresponds to zero data loss ratio (Fig. 7). In this work, for more precise detection, the transceiver output power is reduced to $-26 \mathrm{dBm}$.

At the same time, when the node is going to alarm state it's necessary to ensure that the message have the best chances to be delivered. Relative signal strength versus transmission distance and related data loss ratio are presented in Fig. 7. The transceiver output power in that case is $+8 \mathrm{dBm}$. As it's shown in the figure the reliable service area of the transceiver is 100 meters since in this distance there are no data losses. At this distance the RSSI value is $-70 \mathrm{dBm}$.

Therefore, during alarm data transmission the output power of the transceiver is setted to $+8 \mathrm{dBm}$ which is the maximum output power of the transceiver in its boost mode.

\section{Power Consumption}

As it was said before, the work cycle of the node is 20 seconds. During the cycle the node goes through following stages: the multistage measurement pulse, the data transmission and presence detection and the power saving mode.

The power consumption of the node during the multistage pulse is presented in Fig. 8.

As it's shown in the figure the power consumption value during first stage of the multistage pulse is $320-280 \mathrm{~mW}$. For second stage the value is $170-150 \mathrm{~mW}$. And during fourth stage it's less than $80 \mathrm{~mW}$. The average power consumption for whole multistage pulse is $77 \mathrm{~mW}$ and it's legth is about $0.6 \mathrm{~s}$.

The multistage pulse is followed by data transmission to assure that the wireless connection is established in common situation or to deliver data during alarm mode. The average power consumption in this stage is about $125 \mathrm{~mW}$ for alarm mode (when transceiver output power is $+8 \mathrm{dBm}$ ) and about $70 \mathrm{~mW}$ otherwise. The node power consumption during data transmission is shown in Fig. 9.

The data transmission takes no more than $0.5 \mathrm{~s}$. But if the wireless connection isn't established it takes about $1 \mathrm{~s}$ to rejoin the network. The node power consumption during joining the network is presented in Fig. 10. The average power consumption of the node in this mode is about 70.5 $\mathrm{mW}$.

Since the cycle length is $20 \mathrm{~s}$, the average power consumption for whole cycle is about $8.56 \mathrm{~mW}$ in worst case of an alarm situation while the connection to the network was lost. And the average power consumptions is about 2.66 $\mathrm{mW}$ in general situation when the connection is established.

Providing $900 \mathrm{mWh}$ power supply the autonomous lifetime of the node will be about 105 hours in worst case and about 338 hours in general situation. Assuming that a work day has 8 hours, that's more than 13 work days long autonomous lifetime. Therefore, the average power consumption of the node is low enough for more than two weeks autonomous lifetime.

\section{Conclusion}

In this work the uniform inbuilt wireless sensor node for working conditions monitoring is presented. The node consists of the sensor module (with the catalytic methane sensor, the temperature sensor and the MCU), the ZigBee transceiver, the light indicator and the buzzer. All these pieces are low power, light-weighted and built in to the clothes. The node is able to signalize about employee presence in relation to working facilities, and to monitor the atmosphere for temperature and combustible gases concentration. Due to the average power consumption optimization, the autonomous lifetime of the node is more than two weeks. The reliable service area of data transmission is 100 meters.

\section{REFERENCES}

[1] Stoppa Matteo, Alessandro Chiolerio. "Wearable electronics and smart textiles: a critical review." Sensors 14.7 (2014): 11957-11992. http://dx.doi.org/10.3390/s140711957

[2] Chan Marie, et al. "Smart wearable systems: Current status and future challenges." Artificial intelligence in medicine 56.3 (2012): 137-156. http://dx.doi.org/10.1016/j.artmed.2012.09.003

[3] Sultan Nabil. "Reflective thoughts on the potential and challenges of wearable technology for healthcare provision and medical education." International Journal of Information Management 35.5 (2015): 521526. http://dx.doi.org/10.1016/j.ijinfomgt.2015.04.010

[4] Scataglini, Sofia, Giuseppe Andreoni, and Johan Gallant. "A Review of Smart Clothing in Military." Proceedings of the 2015 workshop on Wearable Systems and Applications. ACM, 2015. http://dx.doi.org/10.1145/2753509.2753520

[5] Pei Zhou, Gongsheng Huang, Linfeng Zhang, Kim-Fung Tsang, Wireless sensor network based monitoring system for a large-scale indoor space: data process and supply air allocation optimization, Energy and Buildings, Volume 103, 15 September 2015, Pages 365374. http://dx.doi.org/10.1016/j.enbuild.2015.06.042

[6] Hang Shen, Guangwei Bai, Routing in wireless multimedia sensor networks: A survey and challenges ahead. Review Article, Journal of Network and Computer Applications, Volume 71, August 2016, Pages 30-49. http://dx.doi.org/10.1016/j.jnca.2016.05.013

[7] A. Somov, A. Baranov, D. Spirjakin, A. Spirjakin, V. Sleptsov, R. Passerone, Deployment and evaluation of a wireless sensor network for methane leak detection, J. Sensors and Actuators A. 202 (2013) 217-225. http://dx.doi.org/10.1016/j.sna.2012.11.047

[8] Alessandro Redondi, Marco Chirico, Luca Borsani, Matteo Cesana, Marco Tagliasacchi, An integrated system based on wireless sensor networks for patient monitoring, localization and tracking, Ad Hoc Networks, Volume 11, Issue 1, January 2013, Pages 39-53. http://dx.doi.org/10.1016/j.adhoc.2012.04.006

[9] Dimitris Kelaidonis, Andrey Somov, Vassilis Foteinos, George Poulios, Vera Stavroulaki, Panagiotis Vlacheas, Panagiotis Demestichas, Alexander Baranov, Abdur Rahim Biswas, Raffaele Giaffreda, Virtualization and Cognitive Management of Real World Objects in the Internet of Things. In: IEEE International Conference on Green Computing and Communications (GreenCom), pp.187-194, IEEE Press (2012). http://dx.doi.org/10.1109/GreenCom.2012.37

[10] Miorandi, D., Sicari, S., De Pellegrini, F., Chlamtac, I.: Internet of Things: Vision, Applications and Research Challenges. J. Ad Hoc Networks 10, 1497-1516 (2012). http://dx.doi.org/10.1016/j.adhoc.2012.02.016

[11] A.Somov, A.Baranov, D.Spirjakin, R. Passerone, Circuit design and power consumption analysis of wireless gas sensor nodes: One-sensor versus two-sensor approach, IEEE Sensors Journal, 14 (6) (2014) 2056- 2063. http://dx.doi.org/10.1109/JSEN.2014.2309001 
[12] Denis Spirjakin, Alexander M. Baranov, Vladimir Sleptsov. "Design of smart dust sensor node for combustible gas leakage monitoring." In Computer Science and Information Systems (FedCSIS), 2015 Federated Conference on (pp. 1279-1283). IEEE. http://dx.doi.org/10.15439/2015F172

[13] Alexander Baranov, Denis Spirjakin, Saba Akbari, Andrey Somov, "Optimization of power consumption for gas sensor nodes: A survey." Sensors and Actuators A 233 (2015) 279-289. http://dx.doi.org/10.1016/j.sna.2015.07.016
[14] Spirjakin D., Baranov A. M., Somov A., \& Sleptsov, V. "Investigation of Heating Profiles and Optimization of Power Consumption of Gas Sensors for Wireless Sensor Networks." Sensors and Actuators A: Physical (2016). http://dx.doi.org/10.1016/j.sna.2016.05.049

[15] Standard GOST R EN 50194-1-2012, Signalizators for the detection of combustible gases in domestic premises, 2000

[16] Standard EN 50194-2000, Electrical apparatus for the detection of combustible gases in domestic premises. Test methods and performance requirements, 2000 . 